\title{
A couples-focused intervention for smoking cessation during pregnancy: The study protocol of the Quit Together pilot randomized controlled trial
}

\author{
Cristian I. Meghea ${ }^{1,2,3}$, Alexandra Brinzaniuc ${ }^{2,3}$, Alexandra Sidor ${ }^{2,3}$, Razvan M. Chereches ${ }^{2,3}$, Dan Mihu4, Cristian I. \\ luhas ${ }^{4}$, Florin Stamatian ${ }^{5}$, Gabriela Caracostea ${ }^{5}$, Marina D. Dasca ${ }^{2,3}$, Kristie Foley ${ }^{6}$, Adriana Baban 7 , Thomas C. Voice ${ }^{8}$, \\ Oana M. Blaga ${ }^{2,3}$
}

\begin{abstract}
Tobacco smoking remains the leading global cause of preventable disease and death. Preconception and pregnancy smoking are high in Central and Eastern Europe. Quit Together is a partnership between a US university and a Romanian university, obstetrics and gynecology clinics in Romania, and other community partners in Romania. The objective of the Quit Together pilot study is to adapt, enhance and test the implementation feasibility and initial efficacy of an evidence-based pregnancy and postnatal couple intervention for smoking cessation in Romania. Quit Together builds on the Motivation and Problem Solving (MAPS) approach, enhanced by targeting the couples' smoking behavior and focusing on dyadic efficacy for smoking cessation. The study is an ongoing randomized controlled trial of 120 Romanian pregnant smokers and their partners. Participants are randomized to: 1) an intervention arm consisting, typically, of up to 8 prenatal and postnatal telephone counseling calls for the women and 4 for their partners, combining motivational strategies and problem-solving/ coping skills to encourage the woman to quit smoking and the partner to support her decision; and 2) a control arm (usual care). The primary outcome is maternal biochemically verified smoking abstinence at 3 months postpartum. Quit Together has the potential to identify effective strategies to increase maternal smoking cessation during pregnancy and smoking abstinence after birth. If effective, Quit Together is expected to have a sustainable positive impact on the health of the child, mother and partner, and potentially reduced health system costs.
\end{abstract}

\begin{abstract}
AFFILIATION
1 Department of Obstetrics, Gynecology and Reproductive Biology, College of Human Medicine, Michigan State University, Michigan, USA 2 Center for Health Policy and Public Health, College of Political, Administrative and Communication Sciences, Babeș-Bolyai University, Cluj-Napoca, Romania 3 Department of Public Health, College of Political, Administrative and Communication Sciences, BabeșBolyai University, Cluj-Napoca, Romania 4 luliu Hatieganu University of Medicine and Pharmacy, Department of Obstetrics and Gynaecology Dominic Stanca Clinic, Cluj-Napoca, Romania 5 Iuliu Hatieganu University of Medicine and Pharmacy, Department of Obstetrics and Gynaecology Clinic I, Cluj-Napoca, Romania

6 Department of Implementation Science, Division of Public Health Sciences, Wake Forest University, North Carolina, USA

7 Department of Psychology, Babeș-Bolyai University, Cluj-Napoca, Romania

8 Department of Civil and Environmental Engineering, College of Engineering, Michigan State University, Michigan, USA
\end{abstract}

\section{CORRESPONDENCE TO}

Oana M. Blaga. Department of Public Health, College of Political, Administrative and Communication Sciences, Babeș-Bolyai University, 7 Pandurilor St, 400376 ClujNapoca, Romania. E-mail: oana.blaga@ publichealth.ro

\section{KEYWORDS}

motivational interviewing, pregnancy smoking, couples intervention

Received: 13 October 2017

Revised: 12 April 2018

Accepted: 13 April 2018

\section{INTRODUCTION}

Cigarette smoking remains the leading global cause of premature and preventable mortality, with most of the 6 million smoking-attributable deaths occurring in low- and middle-income countries - LMIC ${ }^{1}$. In addition to its effects on mortality, women of reproductive age are especially vulnerable due to the effects of smoking on reproduction, pregnancy 
and neonatal health outcomes. Preconception and pregnancy smoking are high in Central and Eastern Europe (CEE). In Romania, preconception smoking is estimated to be close to $30 \%$, with an estimated $15 \%$ of pregnant women continuing to smoke ${ }^{2}$. By comparison, estimates for the USA are $11 \%$ and $8 \%$, respectively ${ }^{3}$.

In addition to high rates of smoking during pregnancy, relapse among pregnant quitters is also high especially when the male partner smokes. In Romania, up to $80 \%$ of smoking pregnant women have partners who are active smokers ${ }^{4-6}$. It is important to address the smoking behavior of couples during the reproductive life. Such interventions can have favorable lifetime effects on the health of the entire family. Pregnancy and birth represent key motivators for receptivity to smoking reduction or cessation for both women and their partners, as evidenced by the spontaneous quit rate. One of the most inhibiting factors for smoking cessation during pregnancy and abstinence after birth is living with a partner who smokes ${ }^{7}$. Consequently, partner's smoking status and support may significantly impact pregnant woman's smoking behavior ${ }^{5}$. Successful prenatal and postnatal smoking cessation and prevention interventions exist $^{8,9}$, but nearly all have been implemented in high-income countries. Most interventions do not include partner support, and the few that did were generally not successful in reducing pregnancy smoking and relapse $\mathrm{e}^{5,10,11}$. Factors potentially limiting the effectiveness of prior interventions include their intensity and delivery method (e.g. the pregnant woman delivered the intervention to the partner), partners' limited knowledge on the health effects of secondhand smoke exposure, and their inability to undertake problem solving with the telephone counselors ${ }^{5}$. Our intervention will test a mutual support-enhancement intervention for the pregnancy quit decision delivered directly to both partners by embedding practical problem-solving strategies within a motivational framework. Local adaptation of smoking cessation and prevention interventions and efficacy/effectiveness high-fidelity trials, conducted in various populations and settings, are needed to increase effectiveness and sustainability of interventions system wide and to translate these into practice and policy with large scale potential effects on public health ${ }^{12,13}$.
This study presents the protocol of the Quit Together clinical trial. The objective of the Quit Together study is to adapt, enhance and test the implementation feasibility and initial efficacy of an evidence-based pregnancy and postnatal couple intervention for smoking cessation in Romania. Quit Together builds on the Motivation and Problem Solving (MAPS) intervention approach, a novel strategy, successful in preventing smoking relapse postpartum in the $\mathrm{US}^{8}$, enhanced by targeting the couples' smoking behavior and focusing on dyadic efficacy for smoking cessation (individual confidence in ability to work with partner, as a couple, to quit smoking $)^{11}$. Recent research has shown that pregnant women in couples with higher dyadic efficacy for smoking cessation were more likely to quit smoking ${ }^{11}$. The randomized controlled trial (RCT) smoking cessation and prevention intervention proposed in this project will begin during pregnancy, will include a postpartum component, and will target women who smoke during pregnancy and their partners.

\section{METHODS}

This clinical trial protocol manuscript followed the SPIRIT 2013 Statement, which provides recommendations for a minimum set of scientific, ethical and administrative elements that should be addressed in a clinical trial protocol ${ }^{14,15}$.

\section{Study setting}

The study began in 2014 and is being implemented in a partnership among Michigan State University, Babeș-Bolyai University in Cluj-Napoca, Romania, and four community partners in Romania: the two largest State-owned obstetrics and gynecology clinics in Cluj-Napoca, part of the Romanian national health system; Novogyn, a private obstetrics and gynecology hospital in the same city, and the Health for Mothers and Infants Association (SAMAS). The clinics and the hospital offer prenatal care and deliver most of the births in the Cluj-Napoca metropolitan area, the second largest city in Romania (population close to 420000 ). The partner clinics and hospital serve the urban population of the city as well as the population from the rural and suburban areas of Cluj County and deliver approximately 6000 births every year. SAMAS Association delivers prenatal education classes in partner medical institutions throughout 
Romania and promotes enrollment in our study through their prenatal educators and promotional materials. Furthermore, we are conducting a continuous enrollment process through online Facebook promotion. This mixed enrollment strategy enables us to reach a large percentage of smoker pregnant women in Romania, enhancing the external validity of the RCT.

The estimated completion date of the study is November 2019. The study was approved by the Michigan State University Institutional Review Board (IRB\# 14-910) and the Ethics Commission at BabeșBolyai University.

\section{Intervention development and cultural adaptation}

The smoking cessation intervention development process used MAPS as the foundational framework. The Motivation and Problem Solving (MAPS) is a counseling approach using a wellness plan to guide the counseling program and substantiates each counselling session on the continuous dynamic shift between motivation enhancement and skill training strategies ${ }^{16}$. MAPS is unique because it addresses substance use within the context of general life stressors and switches continuously between its two components $^{8,16}$. The wellness plan is a treatment plan established collaboratively with the client during the first counseling sessions and adapted throughout the course of the treatment. The intent is to help participants maintain investment in the counseling process, to improve overall wellbeing, and to increase quitting and abstinence success. Besides the smoking behavior, the wellness plan may include other life stressors that clients' may have, such as lifestyle changes, anxiety, depression and interpersonal problems. Motivational Interviewing is used to enhance client's motivation and commitment to quit smoking and stay smoke free.

MAPS has demonstrated efficacy in preventing postpartum smoking relapse in the US ( $42 \%$ MAPS vs $28 \%$ usual care abstinence at 8 weeks postpartum $)^{8}$ and is also being tested for addressing alcohol, diet and physical activity ${ }^{16-18}$. The treatment mechanism used in MAPS ${ }^{16}$ incorporates potential mediators underlying smoking cessation, including motivation $^{19}$, self-efficacy ${ }^{20}$, coping behaviors ${ }^{21}$, perceived stress $^{22}$ and negative effect ${ }^{23}$. These mediators may impact smoking cessation and maintenance not only individually but also through their reciprocal relations ${ }^{24}$.

The intervention development and adaptation, described below, was an iterative process informed by formative qualitative and survey data collection, manuals of prior MAPS interventions, and the prior experiences of the counselors. Fifteen interviews were conducted with pregnant women, both smokers as well as former smokers, to identify areas that might require adaptation of the intervention strategy to the sociocultural context of Romania. The dimensions pursued were the broad context of smoking (identifying high-risk situations, partner smoking behavior, social norms), outcome expectancies (of smoking and quitting smoking), previous quit attempts, attitudes, knowledge and beliefs about smoking during pregnancy, motivation to quit or remain abstinent, coping mechanisms, identity and transition to motherhood in the context of smoking, as well as healthcare system interactions. Pregnant women were recruited while attending prenatal care and invited to participate, and signed a consent permitting the research team to contact them. A member of the research team contacted them via telephone, obtained verbal consent for participation, scheduled a face-to-face interview or administered the interview via telephone. All interviews were audio-recorded with the permission of the participants and transcribed verbatim for analysis. NVivo-assisted thematic analysis was conducted on the data, and results were mapped to highlight sociocultural variations. Results were summarized and used in the development of the instruments for the subsequent survey of smokers and ex-smokers, as well as used in the counselors training.

Additional data were collected through questionnaires from 143 smoker and ex-smoker pregnant women seeking prenatal care in the three clinics included in the study. Two data collectors trained in the study procedures approached women and obtained informed written consent to participate in the study. A printed questionnaire was administered to assess women's sociodemographics, medical and reproductive history, smoking and alcohol consumption, emotional health, and quality of relationship with their partner. In addition, we inquired about women's willingness to participate 
in a potential couples-focused smoking cessation trial and their preferences regarding incentives and evaluation settings. We conducted descriptive and bivariate statistics to describe our sample and to identify differences between smokers and exsmokers on variables of interest such as: education, home smoking rules, number of previous pregnancies and living children, heaviness of smoking, age of smoking onset, number of quit attempts, importance assigned to quitting/staying quit, confidence in quitting/staying quit, partner's smoking status, dyadic efficacy for smoking cessation, dyadic coping, partner interactions, and relationship quality. The objective of this analysis was to inform the intervention development by analyzing interventionrelevant factors associated with smoking cessation during pregnancy.

\section{Participants}

The smoking cessation trial aims to recruit 120 pregnant women in the first 28 weeks of their pregnancy who are self-reported smokers assessed through a multiple-choice question (including: I smoke, but fewer cigarettes than before pregnancy; I smoke once in a while; I smoke, same as before pregnancy). Additional enrollment criteria include age 18 years or older, married or living with a partner, owning a phone, willing to provide phone contact for their partner, and willingness and cognitive ability to understand and sign the informed consent to participate in the trial. Partners are contacted and invited to enroll in the study in the first two weeks after the pregnant women's enrollment. Their verbal consent will be obtained over the phone. In the event that the partner declines participation, the couple will not be able to be included in this trial.

\section{Recruitment of participants}

Recruitment in the randomized controlled trial is ongoing (Figure 1), using recruitment strategies that were successful in our prior studies in the two clinics and in other community based settings in Romania ${ }^{2,25}$ and new strategies described below. Initially, two research assistants (RAs) trained in the study's standard operating procedures approached women who were waiting for a prenatal consultation in the partner clinics, briefly described the purpose of the study, and invited them to participate in the study.
Figure 1. RCT study design

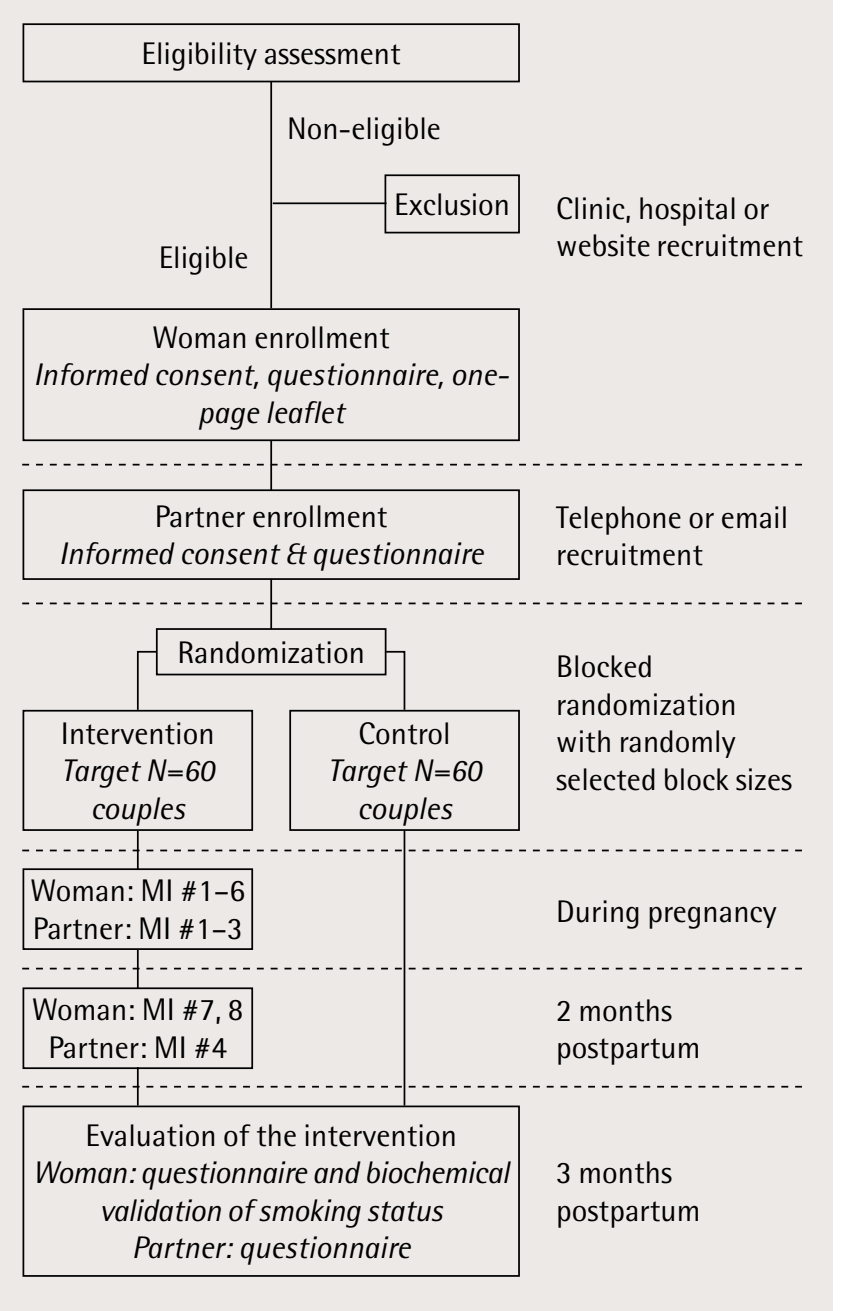

Interested women were provided with details about the trial. The RAs explained that the women's decision to quit is entirely theirs and it does not influence eligibility to participate in the trial, provided details about the enrollment process, the possibility of participating in telephone counselling sessions if randomized in the intervention arm, and the final follow-up to evaluate the intervention, explained the participation risks and benefits, and answered any questions a potential participant may have had. Women were assured that refusal to participate in the study would not influence the care they receive and that they can withdraw from the study at any time.

Those who agreed to participate in the study were asked to sign an informed consent form to participate in the project. Once the informed consent was 
signed, women were invited to complete the selfadministered baseline Quit Together questionnaire, including items on sociodemographics, their reproductive and medical history, tobacco and alcohol consumption, relationship with their partner including partner support with smoking cessation, and emotional health. Women who met the eligibility criteria but declined participation in the study were invited to answer five questions regarding their age, ethnicity, residence, marital status and education. These data will allow the research team to assess participant differences that may potentially bias or limit the generalizability of the intervention effects, as well as to improve and tailor enrollment strategies.

In addition, we expanded our recruitment process through self-enrollment of participants in the RCT via a dedicated project website. The website contains information about the trial, including a help request form, and directs potential participants to an eligibility screener, informed consent, and the Quit Together baseline questionnaire hosted on the secured Qualtrics platform ${ }^{26}$. If the woman is not eligible to participate, a message screen describing the reason for not being eligible is provided. Eligible participants are considered to have given consent if they type their full name and current date in the textbox located at the end of the online informed consent form. Women have the option to download and print their own copy of the consent form. After eligibility and consent procedures, women are asked to provide their contact information and the contact details of their partners, in order for RAs to contact partners over email and/or phone and invite them to participate in the trial. Partners who agree to participate have the option to complete the enrollment process electronically or by phone.

The self-enrollment process is supported by an online and offline project dissemination strategy, enabling us to scale up the clinical trial recruitment and reach national coverage. We developed flyers and posters to advertise the trial and disseminated these in state-owned and private gynecology clinics in Cluj-Napoca and other nearby cities. Promotional materials briefly describe the intervention, include the eligibility criteria for enrollment in the trial, and invite women to visit the RCT's website or to call a local phone number if they want to find out more information about the study. With the help of the Health for Mothers and Infants Association (SAMAS), we are disseminating these promotional materials in SAMAS prenatal education classes and among their partner medical institutions throughout the country. In addition, clinical trial participants are also being recruited via Facebook through targeted advertisements based on: 1) gender (women); 2) age (18-45 years); 3 ) location (Romania); and 4) keywords related to pregnancy, motherhood, expectant parents, and tobacco use. Advertisements contain an image and text consistent with Facebook requirements, and encourage pregnant smokers potentially interested in the RCT to visit the study's website, where they have the option of initiating the self-enrollment process. Recruitment over Facebook has been successfully used in previous smoking cessation trials implemented in high-income countries $^{27,28}$.

\section{Sample size}

The enrollment target is 120 couples, of which 60 couples randomized to the intervention group and 60 couples to the control group. Our primary goal is to establish feasibility and potential efficacy for a future effectiveness RCT. The trial enrolls pregnant smokers in the first or second pregnancy trimester. A 0.2 cessation rate is anticipated in the control group $^{3}$. At two-tailed alpha of 0.05 , our sample of 120 families (60 intervention, 60 control) will yield a power above $80 \%$ to detect superiority of 0.23 or larger in smoking cessation rates in the intervention group.

\section{Randomization}

Participant identification codes have been randomized to the intervention and control arms, using a blocked randomization procedure with randomly selected block sizes (www.randomization. com), in order to ensure size balance across study groups and prevent selection bias ${ }^{29}$. Data collectors allocate identification codes sequentially to enrolled study participants, and both data collectors and study participants are blind to the randomization status. The first author and a research assistant continually monitor enrollment and validate allocation to intervention and control groups, based on the randomization process. 


\section{Interventions}

The control arm is the usual care and the intervention arm is MAPS-based prenatal and postnatal counseling for mothers and their partners, delivered by telephone as described below. All couples, both in the intervention and control arm, receive three $\$ 12$ (50 RON) gift cards by mail, two immediately after recruitment and one after the 3-month postpartum follow-up assessment.

\section{Control arm}

Participants and their partners allocated to the usual care control arm do not receive phone counseling. For ethical reasons, all mothers in both the control and the intervention arms receive a one-page leaflet with information related to smoking cessation, the benefits of staying smoke-free and how the partner could help with smoking cessation. Some of this information may also be provided routinely by the clinics' personnel during the usual prenatal and postnatal care.

\section{Intervention arm}

\section{Interventionists}

The counselors, two females and one male, were gender matched with the clients. All three counselors have a Masters degree in clinical psychology and were previously trained in delivering cognitivebehavioral therapy. Two counselors were accredited in conducting psychotherapy while one was accredited in delivering psychological counseling. Specific to the intervention, they received MI training including workshops led by a certified member of the Motivational Interviewing Network of Trainers, studied the intervention manual in detail and received smoking cessation training. In addition, all counselors practiced delivering the intervention through roleplaying with study investigators and other staff. The counseling sessions were audio-taped and evaluated by a Quit Together research team member supervising counselors' activity. This supervisor, with a BA and MA in psychology and training and expertise in MI, evaluated all counseling sessions to ensure counselors respect not only the intervention manual but also the MI approach. As an additional training tool, the counselors listened to their own counseling sessions. The supervisor delivered the feedback to the counselors face-to-face and via Skype. The counselors further practiced delivering between two and four counseling sessions with male and female adults interested in quitting, and with pregnant smokers and their partners. The supervisor reported to the research team any progress and concerns regarding counselors' skills in delivering the intervention. Counselors and the MI supervisor meet monthly, listen, code, discuss randomly selected sessions and resolve any issues that may occur, in order to ensure intervention fidelity.

\section{Intervention manual}

The development of the intervention manual began during the formative data collection phase and was informed by conversations with the Romanian research team, the counselors and US researchers, including a MAPS expert consultant. The intervention manual, in Romanian, used translated material from current MAPS manuals developed for behavioral interventions in the US, adapted and enhanced to include the focus on pregnant couples and on the dyadic efficacy for smoking cessation. The intervention manual was finalized using information from the intervention pre-testing phase, with active participation of the counselors. The manual comprised: 1) an introduction in the MAPS approach, 2) fundamental knowledge needed to apply MAPS, 3) motivation enhancement information, 4) skill training information, 5) the wellness plan, 6) structure of counseling sessions for women and their partners, 6) instruments for counselors, and 7) information on smoking and on staying smoke free.

The counseling sessions will be based on the intervention manual, which is not intended to be followed as a script. The purpose is rather to utilize general MI and problem-solving techniques, and support the delivery of the intervention through specific modules in the manual, individualizing treatment for each participant. The specific focus of the counseling program may be different for each participant, depending on the concerns, specifics of the smoking behavior, prior quit attempts, partner support and barriers to smoking cessation. Key to the treatment program, however, will be to consider the link between the problems and issues discussed during the counseling sessions and the smoking cessation of the pregnant women, which is the main focus of the trial. 


\section{Structure of counseling sessions}

The counseling program for the pregnant smokers will begin by assessing the smoking history of the pregnant women, including prior quit attempts and the role of their partners, discussing the importance, confidence, and readiness to quit smoking and introducing the wellness plan. The women's counseling sessions focus on establishing smoking cessation and abstinence as goals on the women's wellness plans. The counselors will help the pregnant smoker set wellness plan goals, preferably related to smoking cessation. Subsequent sessions will focus on reassessing and revising the goals, and on the progress towards the goals on the wellness plan.

The partners' sessions focus on both supporting the women to quit smoking and, if a smoking partner indicates an intent to reduce or quit, to also support him in this direction. The counseling program for the women's partners will begin by discussing the prior quit attempts of their wife/partner, mainly how they managed together the smoking cessation process. If the partners smoke, the counselors will attempt to discuss how they could facilitate the wife/ partner's quitting decision (e.g. quitting together, not smoking in the home, not smoking in the wife/ partner's presence). Goals and a wellness plan will be discussed with the partner as well. Subsequent sessions will focus on reassessing and revising the goals, and on the progress towards the goals on the wellness plan, addressing high risk situations for smoking for the wife or partner, and enhancing the dyadic efficacy for smoking cessation, keeping in mind that the main focus of the trial is the pregnant women's smoking cessation. A suggested detailed structure of the counseling sessions for pregnant women is presented in Table 1. A similar structure applies to women's partners focusing on supporting the women in quitting smoking and staying smoke free.

\section{Intervention delivery}

The telephone-based counseling sessions are led by the counselors, specially trained to offer support to quit smoking and typically last 20-30 minutes. Telephone counseling sessions are provided during pregnancy and the first 2 months after birth. A typical counseling program may include up to 8 sessions offered by female counselors to the
Table 1. Suggested structure and content of Quit Together counselling sessions

Session
Session 1
1. Introduction and agenda (establish rapport)
2. Review confidentiality
3. Smoking history, previous quit attempts, and
current smoking
4. Importance/confidence/readiness rulers
5. If the participant is not ready to quit or set a quit
day: Building motivation
a. Decisional balance
6. If the participant is ready to quit: Preparing for
the quit attempt
7. Introducing the wellness plan
8. Ending the session: Schedule next session

Session 2

1. Continue building rapport - Introduce the agenda

2. Review and revise the wellness plan goals

3. Address high-risk smoking situation

4. Complete the values exercise

5. Enhance participant's self-efficacy

6. Enhance dyadic efficacy

7. Schedule next session

Sessions 3-7

1. Continue building rapport - Introduce the agenda

2. Review and revise wellness plan goals

a. Review progress with quitting

b. Review barriers to quitting

c. Review high-risk situations, avoid cope-escape strategies, and lapses.

3. Ruler check-in

5. Decisional balance exercise as needed/indicated

6. Use information modules as needed/indicated

7. Schedule next session

Session 8

1. Reconnect with the participant

2. Review progress

3. Consider next steps

4. Farewell and provide referrals as necessary

pregnant smokers, 6 during pregnancy and 2 after birth, and up to 4 offered by a male counselor to the partners, 3 during pregnancy and 1 after birth. The exact number and frequency of counseling sessions are individually determined by participants and the counselor, depending on the participants' preferences and needs. The individualized approach to the frequency and number of sessions is derived from, and in line with, the most recent MAPS 
interventions ${ }^{16-18}$. A distinct component of the intervention, and a focus of the counseling sessions with both partners, is to enhance both partners' dyadic efficacy for smoking cessation ${ }^{11}$, aiming to improve skills to work together as a team to help pregnant smokers quit. Dyadic efficacy enhancing techniques used by the counselors with both partners will include focusing on activities that the couple do together or building upon prior successes, working as a team towards behavior change.

The protocol specifies a designated time-frame after trial enrollment for the counselors to contact participants for the first counseling session. Subsequent sessions are scheduled by participants and the counselor depending on the participants' preferences and needs. For example, if a pregnant smoker sets a quit date during the first counseling session, the counselor will recommend scheduling the second session on the quit day. If the counselor cannot reach a participant on or around the scheduled session day, the counselor tries to establish contact over several days according to protocol. If unsuccessful in reaching the participants, the counselors will repeat the protocol for attempting another contact once every 4 weeks. All telephone counseling sessions are recorded upon receipt of verbal consent for recording.

\section{Primary outcome measures}

Participant satisfaction measured through a tailored client satisfaction questionnaire will be used to assess feasibility. Other feasibility measures will include uptake rates, adherence/compliance rates, intervention 'dose' (number of counseling sessions), and follow-up rates in the intervention arm and separately among women and their partners. The primary clinical outcome will be the binary maternal smoking abstinence assessed at 3 months after birth $^{28}$. Prolonged abstinence (PA) and 7-day point prevalence abstinence (PPA) will be the primary outcomes assessed at 3 months post-birth. PA failure will be defined as self-reported 7 or more consecutive days of smoking since birth or smoking at least 1 cigarette over the 2 consecutive weeks prior to the assessment. Regarding PPA, participants who report 'no smoking, not even a puff' in the previous 7 days will be mailed a saliva cotinine test and asked to send two photos: one as they give a saliva sample and one of the completed test ${ }^{28}$. Those with a salivary cotinine $<10 \mathrm{ng} / \mathrm{mL}$, will be considered confirmed nonsmokers. Participants reporting electronic nicotine delivery systems (ENDS) use will be recorded and reported separately.

\section{Secondary outcomes}

Secondary outcomes of interest at the final followup will include: number of 24-hour quit attempts since enrollment, the number of smoked cigarettes per day (for those who were not able to quit), the dyadic efficacy for smoking cessation, self-efficacy, smoking-related partner support behaviors, teamwork standards, and dyadic coping reported by the women. Partners will report at the final follow-up: the dyadic efficacy for smoking cessation, PPA and quit attempts if they smoke. To assess the smokers' confidence in their abilities to work together as a team with their partners to quit smoking and cope with quitting challenges, we will use the Dyadic Efficacy for Smoking Cessation instrument ${ }^{11}$. Selfefficacy will be measured using the Romanian version of the General Self Efficacy scale previously translated, adapted and validated in Romania ${ }^{30}$. Three items evoking positive behaviors (items 1, 7 and 9) and three evoking negative behaviors (items 2, 3 and 7) were selected from the Partner Interaction Questionnaire and used ${ }^{31}$ to measure partner support in women's smoking cessation attempts. One item will use a Likert scale (1-10) to measure the extent to which respondents believe issues related to the quit attempts should be handled as a team or individually ${ }^{11}$. An adapted version of the dyadic coping subscale of the Bodenmann Dyadic Coping tool, modified to the cessation context, will be used to assess how the couple together may cope with stress related to quitting smoking ${ }^{11,32}$. Secondary outcomes are all self-reported by women and their partners, measured at RCT enrollment and at the 3-month post-birth follow-up.

\section{Statistical analysis}

To evaluate the efficacy of the intervention, we will initially perform unadjusted comparisons of the outcomes in intervention vs the control groups. We will then use regression analyses to adjust for baseline group dissimilarities (when $\mathrm{p}<0.10$ ). Data quality control will include data accuracy 
checks, outlier examination, missing data patterns, distributional assumptions, and the intervention/ control balance on a variety of characteristics at baseline. We will employ intent-to-treat techniques for all analyses with those lost to follow-up considered continued smoker or relapsed. Separate analyses will be performed for women and partners. To test our primary-treatment effect hypotheses, we will employ multivariate analyses including random effects regression models (subject-specific effects) and the method of generalized estimating equations (population-averaged effects, with fewer assumptions), modeled to include the effect of time with participant. Our papers will report crude and covariate-adjusted efficacy estimates from both models, allowing readers to draw inferences based on both approaches. In addition to the above outlined plans to address potential randomization imbalances, all multivariate analyses will include an indicator for participation in other cessation treatments, to account for possible contamination. We will also perform efficacy sensitivity analyses, excluding the possible contaminated cases, if any.

\section{DISCUSSION}

Quit Together has the potential to identify effective strategies to increase maternal smoking cessation during pregnancy and smoking abstinence after birth. With a focus on the couple, the strategies may be effective to increase smoking cessation and abstinence among both women and their partners as well. The strengths of the intervention include its scientific rigor, the implementation in a developing region of the world with limited access to health resources and cessation services, with high smoking prevalence, and where such interventions are lacking, the couple approach with an emphasis on the dyadic efficacy for smoking cessation, the anticipated contribution to the field of research on smoking cessation during and around pregnancy, and the high potential to be adopted in the universal health system in Romania that includes a quit line, with applications to the US and other populations. If proven effective, all quit-line counselors can be MAPS-trained and deliver proactive phone counseling.

Limitations of the study include potential difficulty enrolling women who have difficult relationships with their partners, the potential low compliance and retention in the study, difficulty to biochemically verify smoking status due to the low likelihood of face-to-face meetings with the new mothers 3 months after birth, and possible missing values in the data available to assess the intervention efficacy, with proposed solutions to address this potential limitation.

The evaluation will report the effects of the Quit Together intervention on: rates of prolonged and point prevalence maternal smoking abstinence biochemically verified via salivary cotinine, number of quit attempts, cigarettes per day among those who continued smoking, maternal motivation, selfefficacy, dyadic efficacy for smoking cessation, paternal smoking abstinence, and cigarettes per day among those who smoke. It will also include mediating and moderating analyses.

\section{CONCLUSIONS}

If effective, Quit Together is expected to have a sustainable positive impact on the cessation and prevention of prenatal and postnatal family smoking, with short- and long-term beneficial health effects for the child, mother and partner, and potential reduced health system costs. This research is also expected to have a significant impact on the methodology and design of clinical and public health interventions during the reproductive years. While the focus of this study is using pregnancy and the reproductive years as a window of opportunity for couple smoking cessation, the approach is relevant for delivering other interventions during the reproductive years, including nutrition, physical activity and child care, with lifetime implications for the entire family.

\section{REFERENCES}

1. World Health Organization. WHO Report on the Global Tobacco Epidemic, 2011. Warning about the Dangers of Tobacco. 2011.

2. Blaga O, Brînzaniuc A, Rus I, Cherecheș R, Wallis AB. Smoking and Smoking Cessation During Pregnancy. An Analysis of a Hospital Based Cohort of Women in Romania. J. Community Health 2017;42(2):333-343. doi:10.1007/s10900-016-0259-6.

3. Curtin SC, Mathews TJ. National Vital Statistics Reports Smoking Prevalence and Cessation Before and During Pregnancy: Data From the Birth Certificate, 2014. 2016.

4. Meghea CI, Rus IA, Rus D. Risk factors associated with nicotine dependence in a sample of Romanian pregnant smokers. Eur. J. Obstet. Gynecol. Reprod. Biol. 
2012;163(1):22-6. doi:10.1016/j.ejogrb.2012.03.033.

5. Hemsing N, Greaves L, O'Leary R, Chan K, Okoli C. Partner support for smoking cessation during pregnancy: a systematic review. Nicotine Tob. Res. 2012;14(7):76776. doi:10.1093/ntr/ntr278.

6. Prady SL, Kiernan K, Bloor K, Pickett KE. Do risk factors for post-partum smoking relapse vary according to marital status? Matern. Child Health J. 2012;16(7):136473. doi:10.1007/s10995-011-0899-1.

7. Schneider S, Huy C, Schütz J, Diehl K. Smoking cessation during pregnancy: a systematic literature review. Drug Alcohol Rev. 2010;29(1):81-90. doi:10.1111/j.1465-3362.2009.00098.x.

8. Reitzel LR, Vidrine JI, Businelle MS, et al. Preventing postpartum smoking relapse among diverse low-income women: a randomized clinical trial. Nicotine Tob. Res. 2010;12(4):326-35. doi:10.1093/ntr/ntq001.

9. Chamberlain C, O’Mara-Eves A, Porter J, et al. Psychosocial interventions for supporting women to stop smoking in pregnancy. Cochrane Database Syst. Rev. 2017. doi:10.1002/14651858.CD001055.pub5.

10. McBride CM, Baucom DH, Peterson BL, et al. Prenatal and postpartum smoking abstinence a partner-assisted approach. Am. J. Prev. Med. 2004;27(3):232-8. doi:10.1016/j.amepre.2004.06.005.

11. Sterba KR, Rabius V, Carpenter MJ, Villars P, Wiatrek D, McAlister A. Dyadic efficacy for smoking cessation: preliminary assessment of a new instrument. Nicotine Tob. Res. 2011;13(3):194-201. doi:10.1093/ntr/ntq236.

12. Spoth R, Rohrbach L, Greenberg M. Addressing core challenges for the next generation of type 2 translation research and systems: The translation science to population impact (TSci Impact). Prev. 2013. doi: 10.1007/s11121-012-0362-6.

13. Storr CL, Cheng H, Alonso J, et al. Smoking estimates from around the world: data from the first 17 participating countries in the World Mental Health Survey Consortium. Tob. Control 2010;19(1):65-74. doi:10.1136/tc.2009.032474.

14. Chan A-W, Tetzlaff JM, Altman DG, et al. SPIRIT 2013 Statement: Defining Standard Protocol Items for Clinical Trials. Ann. Intern. Med. 2013;158(3):200. doi:10.7326/0003-4819-158-3-201302050-00583.

15. Chan A-W, Tetzlaff JM, Gøtzsche PC, et al. SPIRIT 2013 explanation and elaboration: guidance for protocols of clinical trials. BMJ 2013;346:e7586. doi: 10.1136/bmj.e7586

16. Vidrine JI, Reitzel LR, Figueroa PY, et al. Motivation and Problem Solving (MAPS): Motivationally Based Skills Training for Treating Substance Use. Cogn. Behav. Pract. 2013;20(4):501-516. doi:10.1016/j.cbpra.2011.11.001.

17. Thomas JL, Luo X, Bengtson J, et al. Enhancing Quit $\&$ Win contests to improve cessation among college smokers: a randomized clinical trial. Addiction 2016;111(2):331-339. doi:10.1111/add.13144.

18. Correa-Fernández V, Díaz-Toro EC, Reitzel LR, et al. Combined treatment for at-risk drinking and smoking cessation among Puerto Ricans: A randomized clinical trial. Addict. Behav. 2017;65:185-192. doi:10.1016/j.addbeh.2016.10.009.

19. Borland R, Yong H-H, Balmford J, et al. Motivational factors predict quit attempts but not maintenance of smoking cessation: Findings from the International Tobacco Control Four country project. Nicotine Tob. Res. 2010;12(Supplement 1):S4-S11. doi:10.1093/ntr/ntq050.

20. Elshatarat RA, Yacoub MI, Khraim FM, Saleh ZT, Afaneh TR. Self-efficacy in treating tobacco use: A review article. Proc. Singapore Healthc. 2016;25(4):243-248. doi:10.1177/2010105816667137.

21. Kamarck T, Lichtenstein E. Program adherence and coping strategies as predictors of success in a smoking treatment program. Heal. Psychol. 1988;7(6):557. doi: 10.1037/0278-6133.7.6.557

22. Robles Z, Garey L, Hogan J, Bakhshaie J, Schmidt NB, Zvolensky MJ. Examining an underlying mechanism between perceived stress and smoking cessationrelated outcomes. Addict. Behav. 2016;58:149-154. doi:10.1016/j.addbeh.2016.02.022.

23. Carmody TP, Vieten C, Astin JA. Negative Affect, Emotional Acceptance, and Smoking Cessation. J. Psychoactive Drugs 2007;39(4):499-508. doi:10.1080/02791072.2007.10399889.

24. Minami H, Yeh VM, Bold KW, Chapman GB, McCarthy DE. Relations among affect, abstinence motivation and confidence, and daily smoking lapse risk. Psychol. Addict. Behav. 2014;28(2):376-88. doi:10.1037/a0034445.

25. Meghea CI, Brînzaniuc A, Mihu D, et al. A couple-focused intervention to prevent postnatal smoking relapse: PRISM study design. Contemp. Clin. Trials 2015;41:2739. doi:10.1016/j.cct.2015.02.002.

26. Qualtrics. 2005. Available at: http://www.qualtrics.com. Accessed July 7, 2017.

27. Ramo DE, Rodriguez TMS, Chavez K, Sommer MJ, Prochaska JJ. Facebook recruitment of young adult smokers for a cessation trial: Methods, metrics, and lessons learned. Internet Interv. 2014;1(2):58-64. doi:10.1016/j.invent.2014.05.001.

28. Ramo DE, Thrul J, Delucchi KL, Ling PM, Hall SM, Prochaska JJ. The Tobacco Status Project (TSP): Study protocol for a randomized controlled trial of a Facebook smoking cessation intervention for young adults. BMC Public Health 2015;15:897. doi:10.1186/s12889-015-2217-0.

29. Efird J. Blocked randomization with randomly selected block sizes. Int. J. Environ. Res. Public Health 2011;8(1):15-20. doi:10.3390/ijerph8010015.

30. Baban A, Schwarzer R, Jerusalem M. Romanian Version of the General Self-Efficacy Scale, 1996. 1996. Available at: http://userpage.fu-erlin.de/health. Accessed July 4, 2017.

31. Cohen S, Lichtenstein E. Partner Behaviors That Support Quitting Smoking. J. Consult. Clin. Psychol. Coyright 1990;58(3):304-309. Available at: http://psy.cmu. 
edu/ scohen/smoking90.pdf. Accessed July 4, 2017.

32. Bodenmann G. Dyadic coping: A systemic-transactional view of stress and coping among couples: Theory and empirical findings. Eur. Rev. Appl. Psychol. / Rev. Eur. Psychol. Appliquée 1997.

ACKNOWLEDGEMENTS

The authors are grateful to the participants, staff, and the health providers involved in the Quit Together study.

\section{CONFLICTS OF INTEREST}

Authors have completed and submitted the ICMJE Form for Disclosure of Potential Conflicts of Interest and none was reported.

FUNDING

Research reported in this publication was supported by the Fogarty International Center of the National Institutes of Health under Award Number K01TW009654. The content is solely the responsibility of the authors and does not necessarily represent the official views of the National Institutes of Health.

PROVENANCE AND PEER REVIEW

Not commissioned; externally peer reviewed. 